\title{
ORIGINAL
}

\section{LOS REGISTROS DE ENFERMEDADES EN LA INVESTIGACIÓN EPIDEMIOLÓGICA DE LAS ENFERMEDADES RARAS EN ESPAÑA (*)}

Óscar Zurriaga Lloréns (1), Carmen Martínez García (2), Vanessa Arizo Luque (1), Maria José Sánchez Pérez (2), Julián Mauro Ramos Aceitero (3), Maria José García Blasco (1), Maria José Ferrari Arroyo (4), Lilisbeth Perestelo Pérez (5), Enrique Ramalle Gómara (6), Maria Luisa Martínez Frias (7), Manuel Posada de la Paz (4), en nombre de los integrantes de la Red REpIER

(1) Servicio de Estudios Epidemiológicos y Estadísticas Sanitarias. Conselleria de Sanitat. Generalitat Valenciana. Valencia.

(2) Registro de Cáncer de Granada. Escuela Andaluza de Salud Pública. Granada.

(3) Servicio de Epidemiología. Consejería de Sanidad y Consumo. Junta de Extremadura. Mérida

(4) Instituto de Investigación de Enfermedades Raras. Instituto de Salud Carlos III. Madrid.

(5) Servicio de Evaluación y Planificación. Servicio Canario de Salud. Gobierno de Canarias. Santa Cruz de Tenerife.

(6) Servicio de Epidemiología y Prevención Sanitaria. Consejeria de Salud. Gobierno de La Rioja. Logroño.

(7) Centro de Investigación sobre Anomalías Congénitas. Instituto de Salud Carlos III. Madrid.

(*) Este trabajo ha sido financiado, en parte, por la ayuda otorgada por el Ministerio de Sanidad y Consumo en la convocatoria de Redes Temáticas de Investigación Cooperativa (RETIC) para la Red Epidemiológica de Investigación sobre Enfermedades Raras (REpIER), código G03/123, y, en parte, por una ayuda del programa de promoción de la investigación biomédica en ciencias de la salud, para la realización de estudios de investigaciones sobre evaluación de tecnologías sanitarias (convocatoria de 2005), expediente PI05/90228.

\section{RESUMEN}

Fundamento: En el caso de las enfermedades raras existen dificultades para utilizar herramientas como los registros de enfermedades. El objetivo de este trabajo es describir la situación de los registros sobre enfermedades raras en España.

Métodos: Se utiliza la información proporcionada por dos directorios de registros sanitarios españoles elaborados por la Agencia Española de Evaluación de Tecnologías Sanitarias, realizando un estudio descriptivo transversal. Los registros identificados en estos directorios se han clasificado como: 1) «Específicos de enfermedades raras», 2) «No específicos pero con información de enfermedades raras», y 3 ) «No informativos sobre enfermedades raras».

Resultados: En el directorio del año 2000 aparecían 82 registros, de los que el $15,8 \%$ se clasificaron en el grupo 1, mientras que en la revisión de 2005 se identificaron 107 registros, $16,8 \%$ en el grupo 1 . Las principales áreas temáticas de los registros sanitarios del grupo 2 fueron el cáncer, la mortalidad, la psiquiatría y la nefrología. En los directorios no se ha hallado ningún registro genérico de enfermedades raras.

Correspondencia:

Óscar Zurriaga Lloréns. Servicio de Estudios Epidemiológicos y Estadísticas Sanitarias. Conselleria de Sanitat.

C/Micer Mascó 31-33. 46010-Valencia.

Correo electrónico: zurriaga_osc@gva.es

\section{ABSTRACT \\ Disease Registries in the Epidemiological Researching of Rare Diseases in Spain}

Background: The use of tools such as disease registries poses a problem in the case of rare diseases. This study is aimed at describing the current situation concerning rare disease registries in Spain.

Methods: The information provided by two Spanish health registries directories prepared by the Spanish Health Assessment Technologies Agencies are employed, a descriptive cross-sectional study being conducted. The registries identified in these directories has been classified as: 1) «Specific rare diseases» 2) «Unspecific but with information on rare disease» and 3) «Non-informative regarding rare diseases».

Results: The 2000 directory listed 82 registries, $15.8 \%$ of which were classified under Group 1, whilst a total of 107 registries, $16.8 \%$ in Group 1, were identified in the 2005 review. The main health registries in Group 2, by topic, were: cancer, mortality, psychiatry and nephrology. No general rare disease registries were found in the directories. 
Conclusiones: Aunque escasos, existen registros sanitarios en España con información sobre enfermedades raras. Se han identificado áreas temáticas con carencias de registros y también registros o sistemas de información no identificados en las fuentes utilizadas. Es necesario continuar los esfuerzos por mejorar la información disponible sobre las enfermedades raras.

Palabras clave: Enfermedades raras. Sistemas de registros. Prevalencia. Sistemas de información.
Conclusions: Although few in number, health registries do exist in Spain including information on rare diseases. Areas have been identified by topic lacking registries and also information systems or registries unidentified in the sources used. Continuing efforts must be made to improve the information available on rare diseases.

Key words: Rare diseases. Registries. Diseases registries. Prevalence. Information Systems.

\section{INTRODUCCIÓN}

Se consideran Enfermedades Raras (ER), incluidas las de origen genético, aquellas con peligro de muerte o de discapacidad crónica (dependencia permanente del sistema sociosanitario), con problemas añadidos en cuanto a la dificultad diagnóstica (escasa investigación etiológica y/o terapéutica) y cuya prevalencia es menor de 5 casos por 10.000 habitantes en la Comunidad. Esta definición de ER, usada en la Unión Europea ${ }^{1}$, es de naturaleza epidemiológica y el límite de frecuencia para la inclusión bajo esta denominación es arbitrario, de modo que en los EE.UU. está fijado en 7 por 10.000 habitantes y en Japón en 4 por 10.000 habitantes. No se han contabilizado exhaustivamente todas las ER, pero las listas más extensas son las facilitadas por la NORD (National Organization for Rare Disorders $)^{2}$, Orphanet ${ }^{3}$ y por la Oficina de Enfermedades Raras de los NIH (National Institutes of Health $)^{4}$, que recogen entre 3.000 y 6.000 enfermedades de etiologías, características y terapéuticas diversas, que pueden manifestarse tanto en la infancia como en la edad adulta. La mayoría de ellas han sido poco estudiadas y su escaso conocimiento hace que el diagnóstico se retrase en muchas ocasiones, o incluso, que algunas nunca sean diagnosticadas, dificultando la adopción de medidas preventivas o terapéuticas 5 .

Todo ello representa una dificultad añadida para utilizar las herramientas usadas habitualmente en información sanitaria. Una de estas herramientas son los registros de enfermedades.

En Epidemiología se aplica el término Registro al fichero de datos concernientes a todos los casos de una enfermedad particular, o a otras condiciones relevantes de salud, en una población definida, de tal manera que, los casos puedan ser relacionados con la población de base ${ }^{6}$. Los registros definidos así se consideran poblacionales, mientras que se denominan hospitalarios o clínicos, cuando se restringen al ámbito de uno o varios hospitales o sistemas de atención clínica. En los registros de enfermedades de base poblacional la información procede de todos aquellos centros, públicos y privados, en los que se diagnostican y/o tratan los pacientes con una determinada enfermedad. Su principal ventaja es la de disponer de un denominador poblacional, lo que permite calcular la incidencia de la enfermedad en su ámbito $^{7}$. Si, además, se realiza seguimiento de los casos, también se podrá calcular la prevalencia y/o supervivencia. Por tanto, una de sus aportaciones básicas es la de proporcionar una visión de la magnitud de la enfermedad en el área geográfica que abar$\operatorname{can}^{8}$. Esta visión global constituye una exigencia para el control de los sesgos de selección en la realización de estudios epidemiológicos.

En contraposición, el ámbito de los registros hospitalarios se refiere a la propia institución, y la información está limitada a los casos atendidos en la misma, siendo su utilidad fundamentalmente clínica ${ }^{9}$. En ocasiones, los registros hospitalarios pueden estructurarse como una red, en torno a un determinado problema de salud, con la participación de diferentes hospitales que utilizan métodos de trabajo comunes. En esta situación, los casos no se podrán considerar representativos de una población, al carecer de un denominador poblacional. 
Por otro lado, en los centros asistenciales, tanto de Atención Primaria como de Especializada, pueden existir bases de datos clínicas, relacionadas con alguna enfermedad, basadas en el interés de algunos profesionales, lo que hay que diferenciar claramente de un Registro Hospitalario. En ocasiones, se puede establecer un sistema de notificación en relación con un determinado problema de salud, que se centraliza, constituyendo un Registro Central, de gran utilidad, especialmente en el ámbito de las enfermedades poco comunes, por tratarse de datos recogidos con normas homogéneas en torno a una enfermedad específica, lo que facilitará la identificación de casos, habitualmente dispersos, para la realización de estudios multicéntricos ${ }^{10,11}$

En el año 2003, dentro de la convocatoria del Ministerio de Sanidad y Consumo de Redes Temáticas de Investigación Cooperativa, se creó la Red Epidemiológica de Investigación sobre Enfermedades Raras (REpIER $)^{12}$ en la que participan 100 investigadores (epidemiólogos, clínicos, farmacólogos, biólogos, inmunólogos y genetistas) de 16 instituciones de salud pública y hospitales. Uno de los objetivos de REpIER es generar un sistema de información epidemiológico y de servicios sanitarios sobre enfermedades raras, y una de las actividades que se plantea es revisar la utilidad de los registros en este ámbito para tratar de dar respuesta a preguntas tales como ¿Es posible utilizar la metodología de los Registros en el estudio e investigación epidemiológica de las enfermedades raras?

El objetivo de este trabajo es describir la situación de los dispositivos de información sobre Enfermedades Raras en España a partir de dos directorios de registros sanitarios españoles elaborados por la Agencia Española de Evaluación de Tecnologías Sanitarias (AETS), clasificándolos según temática.

\section{MATERIAL Y MÉTODOS}

Se ha realizado un estudio descriptivo transversal, a partir de los dos directorios de registros sanitarios elaborados, en los años 2000 y 2005, por la Agencia Española de Evaluación de Tecnologías Sanitarias ${ }^{13,14}$. Estos directorios son la única base estándar y homogénea existente en España, hasta la fecha, que permita valorar la existencia de registros sanitarios, en el ámbito de las enfermedades raras.

En ambos directorios se ha utilizado como definición de Registro Sanitario la siguiente: «archivo de datos sistemático, continuado y recuperable de manera eficiente relativo a elementos de importancia para la salud, en una población definida, de modo que los elementos registrados puedan relacionarse con una población base».

Los registros o dispositivos de información identificados en estos directorios se han clasificado como: 1) «Específicos de ER», en función de que en su denominación, objetivo o contenidos hagan referencia a una ER según la definición de ER de la Unión Europea; 2) «No específicos pero con información de ER», incluyendo aquellos que no entren dentro de la categoría anterior pero que en función de su denominación, objetivo o contenidos se presuponga, en base a otra información (publicaciones, etc), que pueden contener información sobre alguna ER; y 3) «No informativos sobre ER».

Esta clasificación es excluyente, de tal forma que un registro sanitario no puede clasificarse dentro de varias categorías. La clasificación atendiendo a los criterios mencionados, ha sido efectuada por dos observadores, $\mathrm{y}$, en el único caso en que no ha habido acuerdo, se ha requerido la opinión de un tercer observador.

\section{RESULTADOS}

En el directorio de la AETS del año 2000 aparecían 82 registros, de los que 13 (15,8\%) 
podían considerarse Específicos de ER, mientras que en la revisión publicada en 2005 se identificaron 107 registros, de los que $18(16,8 \%)$ eran específicos de ER (Tabla 1).

Tal como se especifica en el mencionado trabajo, no se incluyeron, en el directorio de registros sanitarios del año 2000, 56 sistemas de información sanitaria relevantes que o bien no cumplían los criterios de inclusión, o bien no se pudo obtener información suficiente para su descripción. Entre ellos se encontraban cuatro que pueden considerarse específicos de ER (de hecho, uno de ellos se incluyó posteriormente en el directorio del 2005): 1) Encuesta sobre Discapacidades, Deficiencias y Minusvalías del INE, 2) Programa de Diagnóstico Neonatal Precoz de Metabolopatías. Dir. Gral. Salud Pública (Ministerio de Sanidad y Consumo). Soc. Esp. Bioquímica Clínica y Patología Mole-

Tabla 1

Fuentes, dispositivos o registros sanitarios españoles específicos de ER

\begin{tabular}{|c|c|c|}
\hline Registro Sanitario & $\begin{array}{l}\text { Directorio } \\
2000\end{array}$ & $\begin{array}{l}\text { Directorio } \\
2005\end{array}$ \\
\hline Registro Nacional de Tumores Cardíacos. & $\mathbf{X}$ & $\mathbf{X}$ \\
\hline Registro Estatal de Lepra. & $\mathbf{X}$ & $\mathbf{X}$ \\
\hline Registro Nacional de la Enfermedad de Creutzfeldt-Jakob. & $\mathbf{X}$ & $\mathbf{X}$ \\
\hline Sistema de Vigilancia de Parálisis Fláccida Aguda en menores de 15 años. & $\mathbf{X}$ & $\mathbf{X}$ \\
\hline $\begin{array}{l}\text { Censo de Pacientes del Ámbito Insalud con Patología de la Hormona del } \\
\text { Crecimiento. }\end{array}$ & $\mathbf{X}$ & $\mathbf{X}$ \\
\hline Estudio Colaborativo Español de Malformaciones Congénitas. & $\mathbf{X}$ & \\
\hline $\begin{array}{l}\text { Registro de Anomalías Congénitas de la Comunidad Autónoma del País Vasco } \\
\text { (RACAV). }\end{array}$ & $\mathbf{X}$ & $\mathbf{X}$ \\
\hline Registro de Defectos Congénitos de la Ciudad de Barcelona. & $\mathbf{X}$ & $\mathbf{X}$ \\
\hline Registro de Defectos Congénitos del Principado de Asturias. & $\mathbf{X}$ & $\mathbf{X}$ \\
\hline Registro de Defectos Congénitos del Vallés. & $\mathbf{X}$ & $\mathbf{X}$ \\
\hline Registro de Mortalidad/Morbilidad de Pacientes del Síndrome del Aceite Tóxico. & $\mathbf{X}$ & $\mathbf{X}$ \\
\hline $\begin{array}{l}\text { Censo de Pacientes del Insalud con Esclerosis Múltiple en Tratamiento con } \\
\text { Interferón Beta (1B). }\end{array}$ & $\mathbf{X}$ & $\mathbf{X}$ \\
\hline $\begin{array}{l}\text { Base de datos del Geltamo (Grupo Español de Linfomas / Trasplante Autólogo de } \\
\text { Médula Ósea). }\end{array}$ & $\mathbf{X}$ & $\mathbf{X}$ \\
\hline Registro de Glomerulonefritis & & $\mathbf{X}$ \\
\hline Registro Nacional de Enfermedades Reumáticas Infantiles & & $\mathbf{X}$ \\
\hline Registro Español de Inmunodeficiencias Primarias (REDIP). & & $\mathbf{X}$ \\
\hline Registro Español de Trasplante en Mieloma Múltiple. & & $\mathbf{X}$ \\
\hline Registro de Pacientes con Esclerosis Múltiple de Cataluña. & & $\mathbf{X}$ \\
\hline Registro Español de Pacientes con déficit de alfa-1-antitripsina. & & $\mathbf{X}$ \\
\hline Total & 15 & 19 \\
\hline
\end{tabular}


Tabla 2

Dispositivos o registros "No específicos de ER pero con información de ER", agrupados por categorías temáticas

\begin{tabular}{|l|c|c|}
\hline \multicolumn{1}{|c|}{ Temática } & $\mathbf{2 0 0 0}$ & $\mathbf{2 0 0 5}$ \\
\hline Cáncer & 17 & 17 \\
\hline Psiquiatría & 9 & 8 \\
\hline Cardiología & 4 & 3 \\
\hline Nefrología & 6 & 11 \\
\hline Ginecología y Obstetricia & 1 & 1 \\
\hline Digestivo & 1 & 0 \\
\hline Mortalidad & 1 & 18 \\
\hline General/Otros & 1 & 1 \\
\hline Total & $\mathbf{3 8}$ & $\mathbf{5 8}$ \\
\hline
\end{tabular}

cular, 3) Registro de Esclerosis Múltiple de Cataluña / Fund. Invest. de la Esclerosis Múltiple, y 4) Registro de Metabolopatías del País Vasco / Consejería de Sanidad Gobierno Vasco».

Dentro de los 82 registros identificados en el año 2000 se encuentran además un total de $40(48,7 \%)$ registros clasificados como «no específicos de ER pero con información de ER». Igualmente, entre los 107 registros sanitarios identificados en el año 2005, 59 $(55,1 \%)$ se clasificaron como «no específicos de ER pero con información de ER» (Tabla 2). En esta categoría se han incluido los registros de tumores infantiles, pues, aunque gran parte de los cánceres en niños son raros no todos lo son.

Las principales áreas temáticas de los registros sanitarios «no específicos de ER, pero con información de ER» fueron el cáncer, la mortalidad, la psiquiatría y la nefrología.
En los directorios de los años 2000 y 2005 se identificaron $29(35 \%)$ y $30(28 \%)$ registros, respectivamente, no informativos sobre ER.

Según la información facilitada por la AETS, todos los registros sanitarios considerados como «Específicos de ER» incluyen una referencia poblacional, y la procedencia de sus datos es de más de una institución o servicio clínico, aunque en más de un caso se trata de registros hospitalarios multicéntricos no poblacionales.

\section{DISCUSIÓN}

Los directorios de registros sanitarios son una herramienta de extraordinaria utilidad para hacer más accesible la información sobre problemas de salud específicos, particularmente en los que, como es el caso de las ER, su baja frecuencia condiciona la dispersión de la información y dificulta su conocimiento. 
Búsquedas genéricas de registros o dispositivos de información como han sido las realizadas por la AETS muestran que, aunque escasos, existen registros sanitarios con información sobre ER, si bien, sólo el 17$18 \%$ del total de los registros sanitarios identificados pueden ser calificados como específicos de ER.

Uno de los objetivos de trabajo planteados en el seno de REpIER, fue la creación de la denominada BADER (Base de Datos de Registros de ER) ${ }^{15}$. La búsqueda de recursos específicos sobre ER dio como resultado la identificación de diferentes tipos de recursos de información. En unos casos se trataba de fuentes o dispositivos de información que no pueden ser considerados registros de población, de acuerdo a las definiciones planteadas anteriormente, sino bases de datos establecidas con una finalidad clínica en un ámbito reducido a un servicio hospitalario, sin embargo, en algún caso se trataba de registros multicéntricos con base en equipos hospitalarios, sin cumplir con las exigencias de exhaustividad de un registro con referencia poblacional. Este es un aspecto sumamente importante en el estudio de las ER, dado que el limitado número de casos dificulta también su utilidad en el ámbito de la salud pública ya que pueden incurrirse en sesgos ${ }^{16}$.

La consideración previa, no implica un cuestionamiento de la utilidad y calidad de las bases de datos clínicas, las cuales, siempre que cuenten con objetivos claros, sean capaces de recoger sistemáticamente y con continuidad datos bien definidos ${ }^{17}$, pueden tener utilidad por su potencial aplicación para la investigación, evaluación asistencial y la gestión de recursos ${ }^{18}$. Por otro lado la sistematización de estas bases de datos permitirá realizar una evaluación de manera estandarizada ${ }^{19}$ y como consecuencia una selección de las mismas basada en criterios de calidad.

En los directorios utilizados no se ha hallado ningún registro genérico de ER y esto plantea la cuestión de si debería existir tal registro. Generalmente los registros se esta- blecen para el estudio de enfermedades que, como es el caso de los tumores, las enfermedades renales o los defectos congénitos, aunque pueden presentar diferencias entre sí en cuanto a su historia natural, tratamiento o pronóstico, también tienen una referencia común. Esto permite que se puedan establecer objetivos comunes para todos ellos y que al menos existan una serie de variables nucleares sobre las que se construye una base común de registro. Entre las conclusiones obtenidas en un inventario de registros realizado en el Reino Unido ${ }^{20}$ se señala que los registros genéricos deben establecerse, y financiarse, sólo si son imprescindibles, es decir si no existe otro medio para alcanzar los objetivos planteados. En el caso de las ER es preciso considerar los problemas de un registro genérico de ER para lograr la exhaustividad necesaria en un marco poblacional. En este sentido, existen dificultades para la localización e identificación de los casos en las correspondientes fuentes de información, que básicamente son debidas a los problemas que plantea el diagnóstico de ER y a la dispersión de los enfermos en múltiples centros públicos y privados, lo que teóricamente incrementaría los recursos necesarios para su gestión. Sin embargo, el reducido número de casos de cada una de estas enfermedades, y la existencia de asociaciones de enfermos, gran parte de ellas agrupadas en la Federación Española de Enfermedades Raras $(\text { FEDER })^{21}$, actuarían como elementos favorables para la creación de un registro. Pero como factor esencial para la valoración de la creación de un registro de ER habría que considerar cual podría ser su aportación para el mejor conocimiento de la magnitud de cada una de estas enfermedades, su distribución espacial, los problemas que plantea su diagnóstico y atención sociosanitaria y en qué medida este conocimiento redundaría en la mejor calidad de vida de este heterogéneo grupo de enfermos y sus familias. En este sentido la pauta de funcionamiento y desarrollo de los registros genéricos de ER la marcarán actuaciones emprendidas recientemente en algunos países europeos, como el 
Registro Nacional de ER italiano ${ }^{22}$, o como la creación del Sistema de Información de ER de Extremadura ${ }^{23}$, éste último surgido en 2004 a iniciativa del nodo extremeño de REpIER. Más recientemente, se ha producido la inscripción en la Agencia de Protección de Datos ${ }^{24}$ del registro de enfermedades raras y banco de tejidos, por parte del nodo de la red REpIER del Instituto de Salud Carlos III. Este registro comenzará a estandarizar sus trabajos tomando como base el esfuerzo desarrollado en la red REpIER y utilizando tecnología que permita un seguimiento a través de Internet. Un ejemplo de intercambio de información ha sido implantado por la propia base de datos Orphanet ${ }^{3}$ donde los pacientes declaran su enfermedad voluntariamente a través de Internet.

A los requisitos necesarios a tener en cuenta en cualquier registro poblacional para iniciar su puesta en marcha ${ }^{20}$ y en el diseño o planificación del mismo ${ }^{25}$ se suman dificultades añadidas en el caso de los registros de ER que hace que se deban tener muy en cuenta no sólo estas recomendaciones sino también que existen otros factores que influyen en el potencial éxito de un registro que deben también ser considerados como son la existencia de un adecuado equipo multidisciplinario, de financiación estable, objetivos claros y liderazgo o prestigio relevante.

En el estudio de la AETS $^{14}$ no se ha evaluado el grado de conocimiento de la existencia de los diferentes registros, pero se afirma que la información sobre registros existentes en nuestro país es poco accesible y escasa. Esto indicaría que la mayoría de los registros a utilizar en el ámbito de las ER son poco conocidos, lo que condiciona, en senti- do negativo, la potencial utilización de la información recogida en los mismos.

Es necesario, asimismo, tener en cuenta que, entre las limitaciones del trabajo efectuado se cuentan la de que los directorios eran generales y no dirigidos de manera concreta a las ER por lo que pueden haberse producido carencias en la identificación. En este sentido, otras búsquedas bibliográficas específicas para ER, complementarias a este trabajo, han puesto de manifiesto registros o sistemas de información no identificados en las fuentes utilizadas ${ }^{26,27}$ y falta de inclusión o desapariciones en alguno de los directorios de algún registro o dispositivo de información en activo $^{28,29}$ lo que evidencia la necesidad de seguir actualizando estos directorios y, en el caso concreto de las ER, de manera específica.

También cabe la posibilidad de que se haya producido un sesgo de información a la hora de clasificar cada registro en una de las categorías debido a que, en ocasiones, la información disponible no permite una clasificación inequívoca del registro.

Por todo ello, creemos que se deben continuar los esfuerzos por mejorar la información disponible en el ámbito de las ER identificando todas las fuentes, dispositivos $\mathrm{y}$ registros que puedan existir, independientemente de su categorización como base de datos clínica o registro poblacional, evaluar posteriormente de una manera homogénea y estandarizada su calidad, adecuación y utilidad para el estudio e investigación de las ER y finalmente plantear qué otros recursos de información serían necesarios para contribuir a la mejor atención de los pacientes con este tipo de enfermedades.

Anexo 1

Integrantes de la red REpIER (Coordinadores de nodos)

Elvira Bel Prieto (GITER, Barcelona), Neus Cardeñosa Marín (Cataluña), Francisco Javier Guillén Enríquez (Andalucía), Gonzalo Gutiérrez Ávila (Castilla-La Mancha), Ma José Iglesias Gozalo (Aragón), Carlos Lahoz Navarro (FJD, Madrid), M José Sánchez Pérez (TMR, EASP, Granada), Ma Luisa Martínez Frías (CIAC, Madrid), Mario Margolles Martins (Asturias), Milagros Perucha González (La Rioja), Manuel Posada de la Paz (IIER, Madrid), Julián Mauro Ramos Aceitero (Extremadura), Ana Robustillo Rodela, Belén Zorrilla Tomás (Madrid), Luis Miguel Ruiz Ceballos (Cantabria), Pedro Serrano Aguilar (Canarias) y Óscar Zurriaga Lloréns (C. Valenciana). 


\section{BIBLIOGRAFÍA}

1. European Commission. Health and Consumer Protection Directorate-General. Useful Information on Rare Diseases from an EU perspective. Luxembourg: European Commission; 2004.

2. National Organization of Rare Disorders (NORD) [citado 15 octubre 2005]. Disponible en: http:// www.rarediseases.org/

3. Orphanet, Paris: INSERM SC 11 [citado 15 octubre 2005]. Disponible en: http://www.orpha.net/

4. Office of Rare Diseases. National Institutes of Health (NIH) [citado 15 octubre 2005]. Disponible en: http://rarediseases.info.nih.gov/

5. Faurisson F. ¿Qué es una enfermedad rara? En: Eurordis, European Organisation of Rare Diseases [citado 15 octubre 2005]. Disponible en: http:// www.eurordis.org/article.php3?id_article $=473$

6. Last JM. A dictionary of epidemiology. New York: Oxford University Press; 1995.

7. Goldberg J, Gelfand HM, Levy PS. Registry evaluation methods. Epidemiol Rev 1980; 2:210-220.

8. Martinez-García C. Registros de enfermedades. Metodología y funcionamiento. Haematologica (ed.esp) 2003; 87(Supl.6):204-207.

9. Young JL. El registro hospitalario de cáncer. En: Jensen OM, Parkin DM, MacLennan R, Muir CS, Skeet RG, (eds). Registros de Cáncer. Principios y Métodos. IARC Publicaciones Científicas No. 95. Lyon: IARC; 1995. p. 173-181.

10. Martinez-Frías ML, Bermejo E, Rodriguez-Pinilla E, Frías JL. Risk for congenital anomalies associated with different sporadic and daily doses of alcohol consumption during pregnancy: a case-control study. Birth Defects Res A Clin Mol Teratol. 2004; 70(4):194-200.

11. SEOP (Sociedad Española de Oncología Pediátrica), Unidad de Documentación Instituto López Piñero. RNTI-SEOP Informe estadístico del Registro Nacional de Tumores Infantiles. Periodo 1980-2002. Libro de ponencias de la XXVI Reunión Nacional de la SEOP; 2003: Palma de Mallorca.

12. Redes Temáticas de Investigación Cooperativa, Instituto de Salud Carlos III: Red Epidemiológica de Investigación en Enfermedades Raras (REpIER). [citado 20 enero 2006]. Disponible en: http://iier.isciii.es/repier/
13. Agencia de Evaluación de Tecnologías Sanitarias (AETS). Instituto de Salud Carlos III. Ministerio de Sanidad y Consumo. Directorio de Registros Sanitarios españoles de utilidad en Evaluación de Tecnologías Sanitarias. Madrid: AETS-Instituto de Salud Carlos III; 2000.

14. Imaz I, Aibar C, González J, Gol J, Gómez-López LI. Características de 107 registros sanitarios españoles y valoración de su utilización. Rev Esp Salud Pública 2005; 79:17-34.

15. Redes Temáticas de Investigación Cooperativa, Instituto de Salud Carlos III: Red Epidemiológica de Investigación en Enfermedades Raras (REpIER). Registros en REpIER. [citado 20 enero 2006]. Disponible en: http://iier.isciii.es/ repier/ html/r_reg.htm

16. Alberman E. Disease registers. En: Armitage P (ed). Encyclopaedia of biostatistics. Vol. II. Chichester (UK):John Wiley; 1998.

17. Pryor D, Califf R, Harrell F, Hlatky M, Lee K, Mark D, Rosatti RA. Clinical databases: accomplishments and unrealized potential. Med Care 1985; 23(5):623-647

18. Black N. Developing high quality databases. BMJ 1997; 315:381-382.

19. Black N, Payne M, on behalf of the DoCDat Development Group. Directory of clinical databases: improving and promoting their use. Qual Saf Health Care 2003; 12:348-352.

20. Newton J, Garner S. Disease register in England. Oxford: Institute of Health Sciences. University of Oxford; 2002.

21. Federación Española de Enfermedades Raras (FEDER). [citado 15 enero 2006]. Disponible en: http://www.enfermedades-raras.org

22. Taruscio D, D’Agnolo G, Greco D. Registro Nazionale delle Malattie Rare. Not Ist Super Sanità 2000; 13(8):1-5.

23. Diario Oficial de Extremadura. Orden de 14 de mayo de 2004 por la que se crea el sistema de información sobre enfermedades raras en la Comunidad Autónoma de Extremadura. DOE núm. 59; 25/05/2004.

24. Boletín Oficial del Estado. Orden SCO/1730/ 2005 , de 31 de mayo, por la que se crean y suprimen ficheros de datos de carácter personal gestionados por el Departamento. BOE núm. 138; 10/06/2005. 
25. Zurriaga O, Bosch A, García-Blasco MJ, Clèries M, Martínez-Beneito MA, Vela E. Aspectos metodológicos de los registros de enfermos renales en tratamiento sustitutivo. Nefrología, 2000; 20(Supl.5):23-31.

26. Giraldo P. Registro español de enfermedad de Gaucher. Haematologica (ed.esp) 2003; 87(Supl.6) 212-216.

27. Gabriel R. Registro español de leucemias (REL). Diseño, metodología, puesta en marcha y resulta- dos preliminares. Haematologica (ed.esp) 2003; 87(Supl.6):216-226.

28. Peris Bonet R. Resultados del Registro Nacional de Tumores Infantiles. An Esp Pediatr 1997, Esp.2:170-172.

29. Martinez-Frias ML, Rodriguez-Pinilla E, Bermejo E; Grupo Periférico del ECEMC. Consumo de tabaco durante el embarazo en España: Análisis por años, comunidades autónomas y características maternas. Med Clin (Barc). 2005 29;124(3): 86-92. 